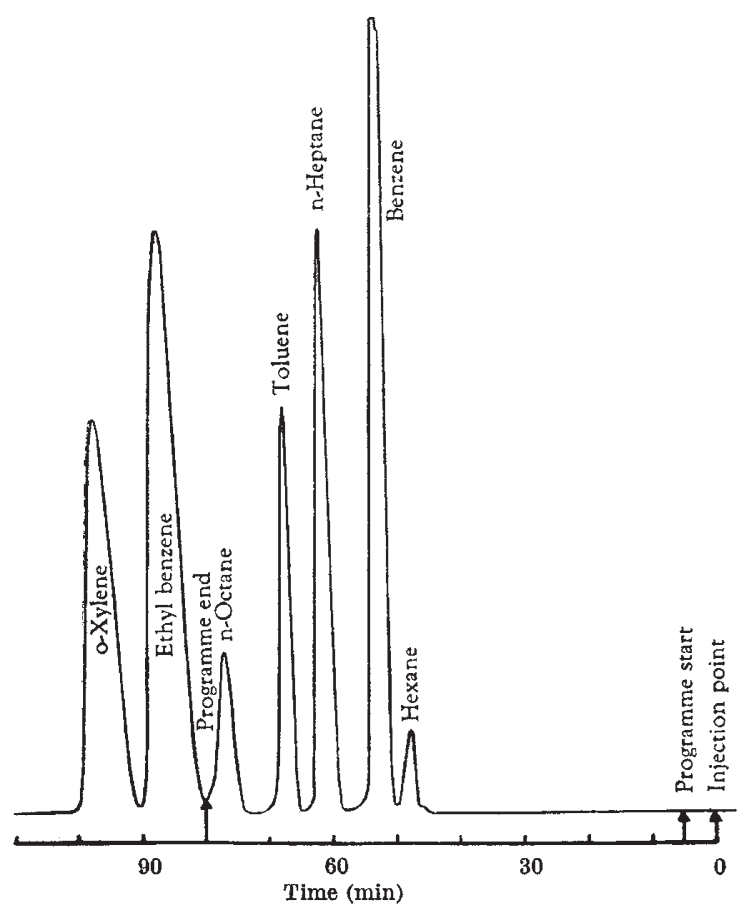

Fig. 1. Chromatograph of a heavily loaded analytical column using programme flow-rate. Size of sample, $175 \mu \mathrm{l}$.; column length, $5 \mathrm{ft}$. colunin diameter, $4 \mathrm{~mm}$; stationary phase, 25 per cent squalane support, $100-120^{\circ} \mathrm{C}$; detector, macro-argon ; chart speed, detector programming time, $75 \mathrm{~min}$; programme flow (exponential), 0-100 ml. mix

obtained. From these results it was predicted that a preparative column of $1 \mathrm{~cm}$ in diameter would be capable of separating $1 \mathrm{ml}$. of the same mixture. In fact, slightly poorer resolution was obtained with this sized charge on the larger column as the efficiency was considerably lower than that of the analytical column.

The assessment of the correct programme for any given mixture is at present being ascertained by experiment, but it has been deduced that the flow programme utilized must be exponential. It should be possible to calculate the correct flow programme from the data obtained when a small sample is chromatographed under normal conditions.

W. G. Pye and Co., Ltd., R. P. W. ScotT Cambridge.

1 Daniel, N. W. R., and Richmond, J. W., Chem. and Indust., 1441 (1961). 2 Scott, R. P. W., Anal. Chem., 35, 481 (1963).

\section{Identification of Purines on Paper Chromatograms}

OwiNG to the frequency with which caffeine is used for the illicit medication of racehorses, the positive identifica tion of $\mu \mathrm{g}$ quantities of this compound is a matter of some importance. Although a caffeine spot on a paper chromatogram exhibits a deep blue fluorescence under ultra-violet light, and may be eluted for subsequent identification by spectrophotometry ${ }^{1}$ or by crystal tests ${ }^{2}$, in common with most other xanthine derivatives caffeine does not react with the usual spray reagents, and there is need of a colour reaction which can be carried out directly on the paper.

Chanda and Chakraborty ${ }^{3}$ have described a method of performing the murexide test on paper chromatograms, but this is not satisfactory on citrate-buffered papers, particularly with compounds isolated from body fluids. We have found that the following method gives good results: The spot is located under ultra-violet light and exposed to bromine vapour for $2 \mathrm{~min}$. In order to avoid the use of large quantities of bromine, this is best carried out by cutting out a narrow strip of paper containing the spot and suspending it over a little liquid bromine in a small conical flask. The paper is next held in the steam over a boiling-water bath for 1 min, then heated for $5 \mathrm{~min}$ in an oven at about $110^{\circ}-120^{\circ}$ C. A rose-pink spot develops, which becomes reddishpurple when exposed to ammonia. Theobromine $(0 \cdot 30)$ and theophylline $(0.55)$ also give this reaction, as do l-n-hexyltheobromine $(0.90)$, proxyphylline $(0 \cdot 72)$, etophylate $(0 \cdot 27)$, and diprophylline $(0 \cdot 43)$, but the differences between the $R_{F}$ values of these compounds, indicated in parenthesis here, and that of caffeine $(0 \cdot 64)$ is sufficient to make differentiation possible. The sensitivity of the test for caffeine is about $10-20 \mu \mathrm{g}$.

\section{E. G. C. Clarke}

S. KaLAYCI

Department of Physiology,

Royal Veterinary College, London, N.W.1.

${ }^{1}$ Moore, P. A., Vet. Rec., 69, 1079 (1957).

${ }^{2}$ Clarke, E. G. C., and Hawkins, A. E., J. Forensic Sci. Soc., 1, 120 (1961).

${ }^{3}$ Chanda, N. B., and Chakraborty, S., Nature, 181, 836 (1958).

\section{BIOPHYSICS}

\section{A Simplified Method for Extraction of Reduced Cytochrome-552 and -556 from Euglena gracilis $(Z)$}

Two spectrally different cytochromes have been isolated by chemical fractionation from Euglena gracilis $(Z)^{x-3}$. Because of the differences in the absorption spectra of the chemically reduced forms, these cytochromes have been designated as cytochrome-552 and cytochrome-556. Cytochrome-552, in the reduced form, extracted from photosynthesizing green cells has absorption peaks at 552,523 , and $416 \mathrm{~m} \mu$, while cytochrome-556, extracted from cells grown in darkness and from Euglena mutants which are non-photosynthetic, has reduced absorption peaks at 556,525 , and $421 \mathrm{~m} \mu$.

The extraction and purification of eytochromes-552 and -556 from Euglena have required large quantities of cells. In addition, the carotenoid and chlorophyll pigments add difficulties to the extraction procedure.

Cytochrome-552 was spectrophotometrically identified in a saline supernatant from an old (38-day) photosynthetic Euglena culture which had been washed with $0 \cdot 9$ per cent sodium chloride $(p \mathrm{H} 6.5)$ in preparation for biochemical extraction. This observation led to question the environmental conditions on the eytochrome extrac. tion. Euglenae gathered at ages 7-40 days, and at $p \mathrm{H}$ 's of $4 \cdot 5-7 \cdot 5$, were extracted in various concentrations of sodium chloride, in $0.05 \mathrm{M}$ phosphate buffer $p \mathrm{H} 7 \cdot 0$, in tap water and in distilled water. All these extractions showed the spectrally reduced form of cytochrome-552. The yield of the extraction was found to be independent of the age, $p \mathrm{H}$, and salt concentration, but dependent on the volume of the extractant.

Since the cytochromes are rolativoly more water-soluble than the carotenoid and chlorophyll pigments, their isolation becomes possible only by using small volumes of water. This method of extraction resulted in obtaining the cytochromes in the spectrally reduced form, whereas. when greater dilutions of water were used, the extracted eytochromes were in the oxidized form.

The procedure for extraction was as follows. From each $300-\mathrm{ml}$. culture bottle in the log phase of growth, lightgrown Euglenae were gathered by centrifugation $(5 \mathrm{~min}$ at $10,000 \mathrm{~g})$. The pelleted cells (1.5 g wet weight) were resuspended, washed, and recentrifuged. This was repeated 3 times, with 40,20 , and $10 \mathrm{ml}$. of 0.9 per cent sodium chloride. No spectrally identifiable cytochrome absorption peaks were evident in any of the 3 discarded supernatant 